\title{
Ohnow Oriput \\ Clinical, biochemical and pathological correlation in alcoholic liver disease among Indian patients
}

\author{
Prasanta Mukhopadhyay, Soumyojit Saha, Cyriac Abby Philips, \\ Uma Sinha
}

\section{Introduction}

Department of General Medicine, Nilratan Sircar Medical College and Hospital,

Kolkata, India

Correspondence:

Dr. Prasanta Mukhopadhyay

Email: drpmookherjee@gmail.com
Alcoholic liver disease is an umbrella term used to describe three important components, namely, fatty liver, alcoholic hepatitis and alcoholic cirrhosis. This hospital based study was conceived to investigate potential correlates between the clinico-biochemical severity and the histopathologic feature of alcohol induced liver disease.

\section{Methods}

A total of 28 consecutive patients suffering from alcoholic liver disease who were admitted to the Department of Medicine, Nilratan Sircar Medical College and Hospital, Kolkata, from January 2009 to August 2010, were enrolled in this study. Patients in whom liver biopsy was contradicted because of co-morbid conditions like coagulopathy, tense ascites and hepatic space occupying lesions, or those who did not consent for biopsy were excluded from the study.

\section{Scoring}

A scoring system was devised to compare and evaluate the correlation and utility of different investigations undertaken in this study. All patients underwent a thorough general examination wherein they were examined for the presence or absence of specific features including icterus, edema, palmar erythema, spider naevi, leukonychia, hair loss, gynecomastia, testicular atrophy, parotid enlargement and Dupuytren's contracture. Each of these signs were assigned a score of one if present and zero if absent.

The abdominal examination of each patient, including physical examination, USG abdomen and upper GI endoscopy were stratified under a separate scoring system. Physical signs that were specifically sought included ascites, presence of prominent superficial abdominal veins flowing away from umbilicus, hepatomegaly and/or splenomegaly. USG abdomen was utilized to look for a dilated portal vein (diameter more than $12 \mathrm{~mm}$ ), portal vein thrombosis and hepatic space occupying lesions. A Doppler ultrasound in parallel was used to examine for altered respiratory phasicity in portal vein flow indicating portal hypertension. Each of the above parameters was given a score of one when present and a score of zero when absent.All patients were subjected to a battery of biochemical tests, which were stratified into a third scoring system. These parameters and their respective scores were as follows. Hemoglobin was measured in all patients and the presence of anemia was given a score of one, while its absence was scored zero. Likewise a platelet count below 1,50,000/cumm was given a score of one. The aspartate aminotransferase / alanine aminotransferase (AST/ALT) ratio was 
ascertained for all patients and was given a score of one if the ratio was $>1$, a score of two if $>2$ and a score of three if $>3$. Prothrombin time was measured for all and was scored as one when raised by 1-3 seconds, two if raised by 4-6 seconds, and three if raised by $>6$ seconds. A low serum albumin $<3.5 \mathrm{gm} \%$ was assigned a score of one. Similarly serum bilirubin was measured in all patients and the parameter scores were derived in line with the Child-Pugh-Turcott score. Hence bilirubin levels were assigned a score of one if the levels were between 1-2 $\mathrm{mg} \%$, a score of two if between $2-3 \mathrm{mg} \%$ and a score of three if bilirubin levels were $>3 \mathrm{mg} \%$.All three scores from the patient's general survey, abdominal examination (including ultrasound and endoscopy) and biochemistry were combined to obtain a clinico-biochemical score for each patient.All 28 patients underwent a percutaneous liver biopsy and were examined for histopathology evidence of alcoholic liver disease under three broad categories. These included: a) fatty liver - centrilobular and perivenular fatty infiltration marked by presence of intracytoplasmic vacuoles; b) alcoholic steatohepatitis ballooning degeneration of hepatocytes along with polymorphonuclear leucocyte infiltration in hepatic lobules; and c) cirrhosis of liver - characterized by varying degree of perisinusoidal fibrosis with regenerating nodules surrounded by thick bands of fibrosis and disordered architecture with pseudo-lobule formation. The presence of each of the above three categories was assigned a score of one, two and three respectively.Simultaneously a Child-Pugh-Turcott scoring was also done for each patient based on available data on ascites, encephalopathy (from history on admission), bilirubin, albumin, and prothrombin time.History of alcohol intake was recorded for each patient specifically as per the duration of alcohol intake including country liquor. Patients were assigned scores of one, two and three for durations of alcohol intake as less than 5 years, 5-10 years and more than 10 years, respectively.

\section{Analysis}

All above scores were subjected to statistical comparison and analysis using the SPSS v14.0 statistical package (SPSS, IBM). Regression analysis was performed keeping histology of liver as the dependent variable and all other parameters including general survey; abdominal examination including ultrasound abdomen, portal vein Doppler ultrasound and upper GI endoscopy; laboratory parameters including hemoglobin, platelet count, transaminase ratio, bilirubin, albumin, prothrombin time; combination of clinical and biochemical parameters; Child-Pugh-Turcott score; and alcohol intake, as independent variables. The regression analysis was aimed to evaluate if liver histology of an alcoholic patient could be reliably predicted by clinical examination of the patient along with any significant correlation with common laboratory investigations, radiological tests, endoscopic examination, history of alcoholism and a combination of all of the above. Therefore, liver histology was taken as the dependent variable.

\section{Results}

Of the 28 patients investigated in this study, $57 \%$ (16) revealed stigmata of chronic liver disease (CLD) $57 \%$ patients gave a history of 150 to $200 \mathrm{ml}$ alcohol intake for more than ten years. $46 \%$ patients revealed a high degree of biochemical abnormalities in the form of hypoalbuminemia in $64 \%$, low platelet count in 53\%, an AST:ALT ratio > 3 times in $17 \%$ and raised prothrombin time in $14 \%$. Based on histopathology $50 \%$ of the patients had liver cirrhosis. Of the remaining 50\%, 15\% had alcoholic steatohepatitis and $85 \%$ had steatosis. Of the patients with CLD and ascites , 31\% showed features of early cirrhosis without portal varices and $18 \%$ had alcoholic hepatitis, with the rest falling into CLD with portal hypertension. So in retrospect, clinical evaluation and histopathological correlation yielded nearly similar results when it came to triaging patients into the three categories of ALD.

It was found that the severity of histopathology changes observed on liver biopsy (steatosis, steatohepatitis and cirrhosis) correlated significantly with the abdominal parameters, with a Pearson correlation coefficient of 0.819 . Of these, hepatomegaly and jaundice correlated best with steatohepatitis. A significant correlation was found for disease severity when measured based on liver histopathology and the combined clinico-biochemical score, with a Pearson correlation coefficient of 0.798 . This suggests that features of tender hepatomegaly when taken along with biochemical features of hepatitis tend to demonstrate a better correlation with features of steatohepatitis. No significant correlations could be drawn between liver histopathology and general survey parameters or between histopathology and various biochemical parameters. Hepatomegaly was the main parameter that correlated best with steatohepatitis. Ascites correlated best with early onset cirrhosis as compared to alcoholic hepatitis, even though both were nearly equally ( $n=5$ vs. $n=3$ ) distributed in these subset of patients. Further, we found a marginal correlation between the clinico-biochemical 
parameters in early compensated cirrhosis without esophageal varices and the abdominal parameters. In this scenario, liver biopsy histology remains the best investigative modality to identify the stage of disease. Regression analysis of our results showed that only abdominal parameters and not biochemical measures were able to predict the severity of histological abnormality in any given case of alcoholic liver disease.

\section{Discussion}

In order to correlate clinical and biochemical findings with histology of liver, a systematic scoring system was devised to evaluate and compare available data from various investigations conducted on our patients. The scoring system was rendered as simple as possible to ensure feasibility of assessment of severity of clinical presentation and biochemical parameters within the resources available in our hospital set up.

The Dionysos study ${ }^{1}$ showed that the incidence of cirrhosis in alcoholic patients was much lesser than previously believed. But the study did not utilize liver biopsy for confirming histological staging in all its patients, which remains the gold standard for assessing the severity of ALD. Another study from Mumbai noted that among 327 patients that were followed up, $41 \%$ had cirrhosis while the remaining $31 \%$ had noncirrhotic liver disease. ${ }^{2}$ This study too did not utilize liver biopsy for confirming the diagnosis in its patients. The results of our study are nevertheless comparable with the latter study.

This study was an attempt to assess the severity of ALD in a set-up with limited resources using percutaneous liver biopsy. We investigated all our study patients on three lines, including clinical, biochemical and histopathological. We found significant correlation between clinico-biochemical severity and liver biopsy changes. Abdominal parameters of hepatomegaly and jaundice correlated best with the stage of steatohepatitis. Ascites correlated best with cirrhosis in early stage, without varices than in those patients with alcoholic hepatitis. Both clinico-biochemical severity and histopathological changes showed no bearing with the duration of alcohol intake even though earlier studies have demonstrated definite correlation with both the amount and duration of alcohol intake.

The limitations of this study were: a) small cohort b) severe cases where percutaneous liver biopsy could not be done were not enrolled and c) recall bias for the duration of alcohol intake Larger studies can examine and substantiate our findings, which if found reproducible can be utilized to assess ALD severity based on simple clinico-biochemical parameters rather than an invasive liver biopsy. Such a protocol/assessment tool becomes all the more pertinent in clinical settings where liver biopsy is either not available or feasible, and yet ascertaining the severity of liver disease is essential for appropriate management of the patient.

\section{References}

1. Bellentani S, Saccoccio G, Costa G, Tiribelli C, Manenti F, Sodde $\mathrm{M}$, et al. Drinking habits as cofactors of risk for alcohol induced liver disease. The Dionysos Study Group. Gut. 1997; 41:845-50.

2. Narawane NM, Bhatia S, Abraham P, Sanghani S, Sawant SS. Consumption of 'country liquor' and its relation to alcoholic liver disease in Mumbai. J Assoc Physicians India. 1998;46:510-3. 